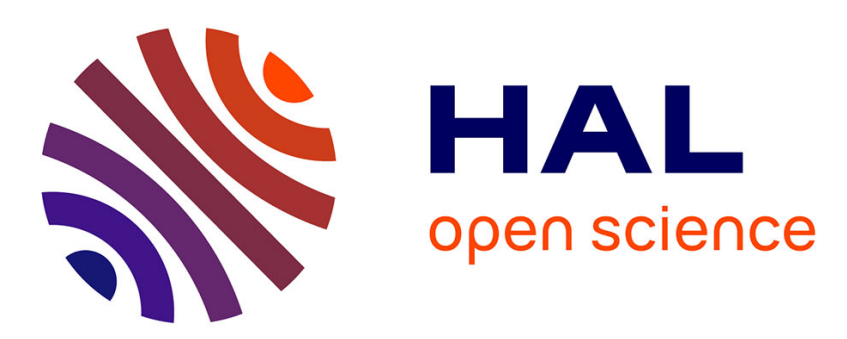

\title{
Marking optimization of stochastic timed event graphs using IPA
}

\author{
Jean-Marie Proth, Nathalie Sauer, Yorai Wardi, Xiaolan Xie
}

\section{To cite this version:}

Jean-Marie Proth, Nathalie Sauer, Yorai Wardi, Xiaolan Xie. Marking optimization of stochastic timed event graphs using IPA. [Research Report] RR-1856, INRIA. 1993, pp.28. inria-00074816

\section{HAL Id: inria-00074816 https://hal.inria.fr/inria-00074816}

Submitted on 24 May 2006

HAL is a multi-disciplinary open access archive for the deposit and dissemination of scientific research documents, whether they are published or not. The documents may come from teaching and research institutions in France or abroad, or from public or private research centers.
L'archive ouverte pluridisciplinaire HAL, est destinée au dépôt et à la diffusion de documents scientifiques de niveau recherche, publiés ou non, émanant des établissements d'enseignement et de recherche français ou étrangers, des laboratoires publics ou privés. 


\title{
Marking optimization of stochastic timed event graphs using IPA
}

\author{
Jean-Marie PROTH \\ Nathalie SAUER \\ Yorai WARDI \\ Xiaolan XIE

\section{$N^{\circ} 1856$} \\ Février 1993
}

PROGRAMME 5

Traitement du Signal,

Automatique et

Productique

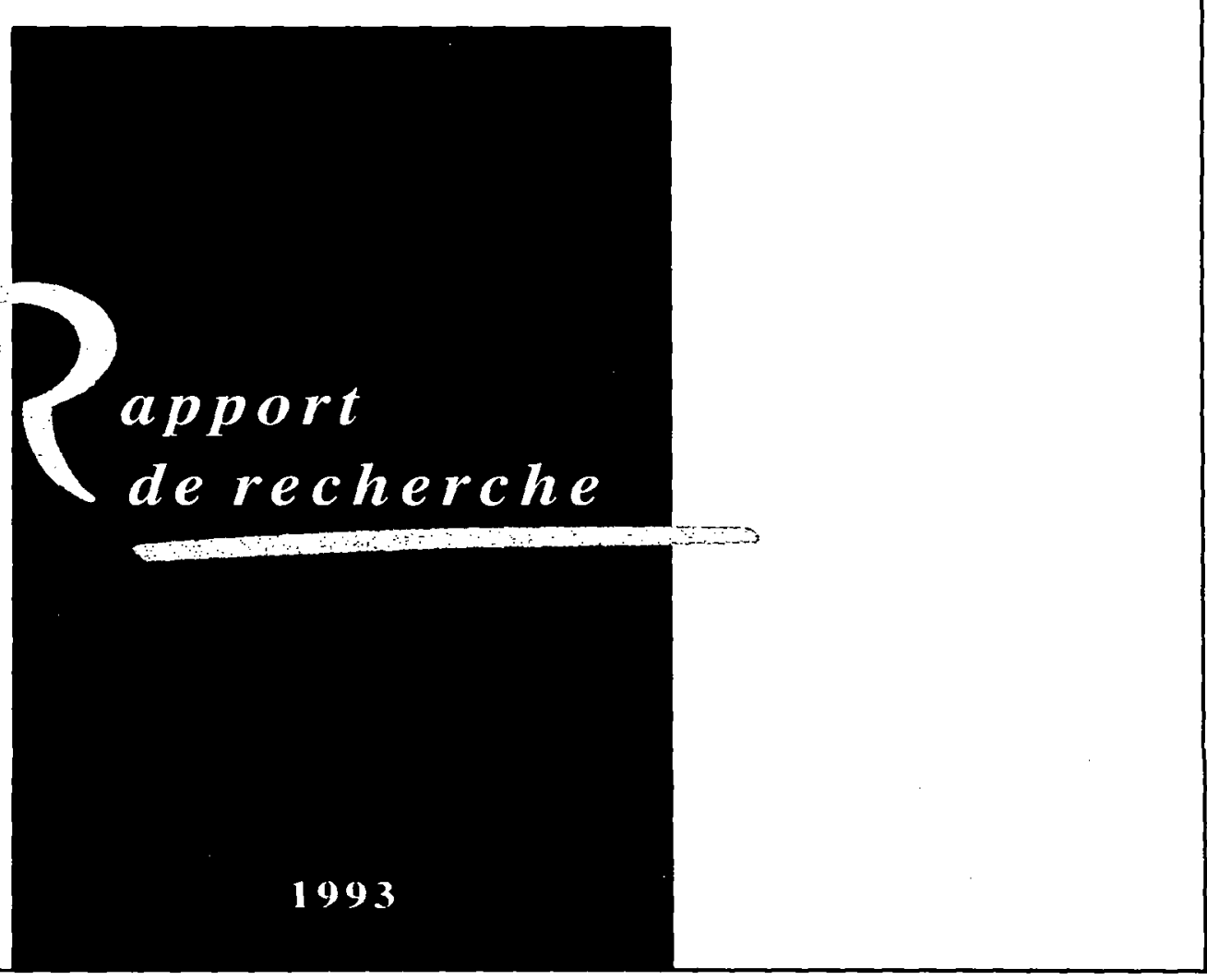




\title{
Optimisation du marquage des graphes d'événements stochastiques à l'aide de l'analyse perturbationnelle *
}

\author{
Jean-Marie PROTH ${ }^{1}$, Nathalie SAUER ${ }^{1}$, Yorai WARDI ${ }^{2}$ et Xiaolan XIE ${ }^{1}$
}

\section{RESUME}

Dans ce papier, nous nous intéressons à l'optimisation du marquage des graphes d'événements stochastiques. Les temps de franchissement des transitions sont générés par des variables aléatoires de distribution quelconque. L'optimisation du marquage consiste à obtenir un temps de cycle donné tout en minimisant un critère p-invariant fonction du marquage initial. Nous montrons d'abord des propriétés importantes de ce problème. En particulier, nous montrons que le temps de cycle d'un graphe d'événements est non-croissant par rapport au marquage initial alors que le critère $p$ invariant est non-décroissant. Nous montrons également que le critère p-invariant de la solution optimale est non-croissant par rapport aux temps de franchissement au sens stochastique. En se basant sur les bornes existantes du temps de cycle, nous montrons que le critère p-invariant devient minimal lorsque les temps de franchissement sont remplacés par leur espérance. Nous établissons également les conditions nécessaires et suffisantes d'atteignabilité d'un temps de cycle donné. Finalement, nous proposons une heuristique pour résoudre le problème d'optimisation du marquage. Cette heuristique part de la solution optimale pour le graphe d'événements déterministe et ajoute successivement des jetons dans des places jusqu'à l'obtention du temps de cycle donné. Nous utilisons la technique de l'analyse des perturbations (IPA) pour identifier les places où ajouter de nouveaux jetons. Les résultats numériques montrent que cette heuristique donne des solutions proches de l'optimum.

MOTS-CLES: Graphes d'événements stochastiques, Systèmes dynamiques à événements discrets, Optimisation du marquage, Propriétés, Analyse perturbationnelle, Algorithmes

(1) INRIA, Technopôle Metz 200, 4 rue Marconi, 57070 Metz, France

(2) School of Electrical Engineering, Georgia Institute of Technology, Atlanta, GA 30332, U.S.A.

\footnotetext{
* Mailing address : Dr XIE Xiaolan, SAGEP Project/INRIA, Technopôle Metz 2000, 4 rue Marconi, 57070 Metz, France. E-mail : xie@ilm.loria.fr Fax : (33) 87763977 Phone : (33) 87203500
} 


\title{
Marking Optimization of Stochastic Timed Event Graphs using IPA *
}

Jean-Marie PROTH 1, Nathalie SAUER 1, Yorai WARDI 2 and Xiaolan XIE 1

\begin{abstract}
This paper addresses the marking optimization of stochastic timed event graphs. The transition firing times are generated by random variables with general distributions. The marking optimization problem consists of obtaining a given cycle time while minimizing a p-invariant criterion. Some important properties have been established. In particular, the average cycle time is shown to be non-increasing with respect to the initial marking while the p-invariant criterion is non-decreasing. We further prove that the criterion value of the optimal solution is non-increasing in transition firing times in stochastic ordering's sense. Based on some existing lower bounds and upper bounds of the average cycle time, we show that the p-invariant criterion reaches its minimum when the firing times become deterministic and we establish the reachability conditions of a given cycle time. We also propose a heuristic algorithm. It starts from the optimal solution to the deterministic case and iteratively adds tokens to adequate places as long as the given cycle time is not obtained. Infinitesimal perturbation analysis of the average cycle time with respect to the transition firing times is used to identify the adequate places in which the new tokens are added. Numerical results show that the heuristic algorithm provides near optimal solutions.
\end{abstract}

KEYWORDS: Stochastic Timed Event Graphs, Discrete Event Dynamic Systems, Marking Optimization, Properties, Infinitesimal Perturbation Analysis, Algorithms

(1) INRIA, Technopôle Metz 200, 4 rue Marconi, 57070 Metz, France

(2) School of Electrical Engineering, Georgia Institute of Technology, Atlanta, GA 30332

\footnotetext{
* Please address all correspondence to Dr XIE X.L.
} 


\section{INTRODUCTION}

Petri nets have been proven to be a powerful tool for modeling complex dynamic systems and evaluating their performances. It provides an unified language for system modeling, property checking and performance evaluation for dynamic systems with synchronization, concurrency and common resources. Successful application areas include communication systems, computer systems, manufacturing systems, etc. Excellent surveys can be found in [13, 22].

This paper is part of our work which intends to provide tools for optimizing the marking of the Petri net model of a real-life system. To better understand the problem, let us consider a manufacturing system. The marking of its Petri net model corresponds to the resources (transportation resources, machines and tools for example) and the work-in-process. Roughly speaking, the marking optimization is equivalent to the optimal utilization of the manufacturing resources.

In this paper we limit ourselves to stochastic timed event graphs in which the firing times are generated by random variables. An event graph, also called marked graph, is a Petri net in which each place has exactly one input transition and one output transition and the weights associated to the arcs are equal to 1 . A strongly connected event graph has some important properties, specifically: (i) the number of tokens in any elementary circuit remains constant whatever the transition firings, and (ii) the system is deadlock free iff each elementary circuit contains at least one token (see for instance $[5,6,9])$.

In the deterministic timed event graph case, it has been proven $[5,16]$ that: (i) the cycle time of an elementary circuit is given by the ratio of the sum of the firing times of the transitions belonging to the circuit by the number of tokens in the circuit; (ii) the cycle time of a strongly connected event graph is equal to the greatest cycle time among the ones of all the elementary circuits. Furthermore, a specified cycle time being given, algorithms have been proposed in [12] to find an initial marking which leads to a cycle time less than the specified cycle time while minimizing a linear criterion function of the initial marking.

In the stochastic timed event graph case, it is no more possible to take advantage of the elementary circuits to evaluate the behaviour of the event graph and to reach a given performance. Previous work mainly focused on ergodicity conditions and performance bounds. Ergodicity conditions have been obtained for timed event graphs [1], for stochastic timed Petri nets [8] and for max-plus algebra models of stochastic discrete event systems [17]. 
For a strongly connected stochastic timed event graph, it has been proven that an average cycle time exists under some fairly weak conditions (see section 2). Both upper bounds and lower bounds have been proposed (see $[2,3,4,14,15,19,25]$ ). In particular, tight upper bounds and lower bounds have been obtained by using stochastic comparison properties (see [3]), superposition properties (see [25]) and large deviation theory (see $[2,19])$. Asymptotic properties with respect to the number of transitions, the transition firing times and the number of tokens in a place were established in [19].

The marking optimization problem for strongly connected stochastic timed event graphs, which consists of obtaining a specified cycle time while minimizing a linear criterion depending on the initial marking, has been addressed in $[15,20]$. It was proven that any cycle time greater than the maximal mean transition firing time can be reached provided that enough tokens are available. Heuristic algorithms for solving the stochastic marking optimization problem have been proposed for solving the normal distribution random variable case in [20] and for solving general case in [15]. However the heuristic solution proposed in [15] starts from the optimal solution to the deterministic case and iteratively adds tokens until the given cycle time is obtained. At each iteration, it computes the ratio of the sum of the mean transition firing times of transitions of each elementary circuit to the number of tokens in it. A new token is added to a place belonging to the circuit with the maximal ratio. This approach has two shortcomings. First, the number of elementary circuits becomes very large as the number of places and transitions increases. Secondly, this approach only uses the first moments of the firing times to determine the places in which the extra tokens are added.

The purpose of this paper is to present new properties of the stochastic marking optimization problem, and to propose a new algorithm for computing near-optimal solutions.

To this end, we give in Section 2 the formal definition of the marking optimization problem of stochastic timed event graphs which consists of obtaining a given cycle time while minimizing a p-invariant criterion. An illustrative example and some possible applications in the field of manufacturing systems are given in Section 3.

Some important properties are proposed in Section 4. In particular, we show that mutually reachable markings have the same average cycle time and the same pinvariant criterion value. The average cycle time is shown to be non-increasing with respect to the initial marking while the p-invariant criterion is non-decreasing. We further prove that the criterion value of the optimal solution is non-increasing in transition firing times in stochastic ordering's sense. Based on some existing lower bounds and upper bounds of the average cycle time, we show that the p-invariant 
criterion reaches its minimum when the firing times become deterministic and sufficient conditions under which the solution to the deterministic case remains optimal are given. We also establish the reachability conditions of a given cycle time.

Section 5 presents a heuristic algorithm. It starts from the optimal solution to the deterministic case and iteratively adds tokens to adequate places as long as the given cycle time is not obtained. Infinitesimal perturbation analysis of the average cycle time with respect to the transition firing times is used to identify the adequate places in which the new tokens are added. This approach avoids the computation of all the elementary circuits at each iteration and, by making use of the sample gradient estimation, can result in very efficient algorithms. Unbiasedness and strong consistency of the Infinitesimal perturbation analysis estimates are established in Section 6. Numerical results presented in Section 7 show that the heuristic algorithm is efficient in providing near optimal solutions.

\section{NOTATIONS, ASSUMPTIONS AND PROBLEM SETTING}

Let $\mathrm{N}=(\mathscr{P}, \mathrm{T}, \mathrm{F})$ be the strongly connected event graph considered. $P$ is the set of places, $\mathrm{T}$ is the set of transitions, and $\mathrm{F} \subseteq(P \times \mathrm{T}) \cup(\mathrm{T} \times P)$ is the set of directed arcs connecting places to transitions and transitions to places. We denote by $\mathcal{M}_{0}$ the initial marking of N.

We assume that no transition can be fired by more than one token at any time (i.e. recycled transitions). This implies that there is a self loop place with one token related to each transition, i.e. $(t, t) \in P$ and $\mathcal{M}_{0}((t, t))=1, \forall t \in T$ where $(t, s)$ indicates the place connecting transition $t$ to transition $s$. We further assume that, when a transition fires, the related tokens remain in the input places until the firing process ends. They then disappear and one new token appears in each output place of the transition.

As a result, the set of places $P$ can be written as $P=\mathrm{P} \cup \mathrm{Pt}$ where $\mathrm{Pt}$ denotes the set of self loop places and $\mathrm{P}$ the other places, i.e. $\mathrm{Pt}=\{(\mathrm{t}, \mathrm{t}), \forall \mathrm{t} \in \mathrm{T}\}$ and $\mathrm{P} \cap \mathrm{Pt}=\varnothing$. Furthermore, since there is always exactly one token in each place belonging to $\mathrm{Pt}$, only the marking of the places belonging to $\mathrm{P}$ will be considered in the following.

Since $\mathrm{N}$ is an event graph, each place has exactly one input transition and one output transition. Without loss of generality, we assume that there exists at most one place between any two transitions. The following notations will be used :

${ }^{\bullet} t$ (resp. $\left.t^{\bullet}\right)$ : set of input (resp. output) places of transition $t$

- $p$ (resp. $\mathrm{p}^{\bullet}$ ) : unique input (resp. output) transition of place $p$ in $(t)$ : set of transitions which immediately precede transition $t$, i.e. 


$$
\text { in }(\mathrm{t})=\left\{\mathrm{s} \in \mathrm{T} / \exists \mathrm{p} \in \mathrm{P}, \bullet \mathrm{p}=\mathrm{s} \text { and } \mathrm{p}^{\bullet}=\mathrm{t}\right\}
$$

out $(t)$ : set of transitions which immediately follow transition $t$, i.e.

$$
\operatorname{out}(t)=\left\{s \in T / \exists p \in P, \bullet p=t \text { and } p^{\bullet}=s\right\}
$$

$(t, s)$ : place connecting transition $t$ to transition $s$

$\Gamma$ : set of elementary circuits of $\mathrm{N}$

$\mathrm{M}_{0}$ : initial marking of the places belonging to $\mathrm{P}$

$\mathrm{M}_{0}(\gamma)$ : total number of tokens contained initially in $\gamma \in \Gamma$

$\mathrm{R}\left(\mathrm{M}_{0}\right)$ : the set of markings reachable from $\mathrm{M}_{0}$

The following notations related to the transition firing times are used throughout this paper :

$X_{t}(k)$ : non-negative random variable which generates the time required for the $k$-th firing of transition $t$

$S_{t}(k)$ : instant of the $k$-th firing initiation of transition $t$

By convention, $X_{t}(k)=0, \forall \mathrm{k} \leq 0$ and $S_{t}(k)=0, \forall k \leq 0$. As shown in [5], the transition firing initiation instants can be determined by the following recursive equations :

$$
\mathrm{S}_{\mathrm{t}}(\mathrm{k})=\underset{\tau \in \operatorname{in}(\mathrm{t})}{\operatorname{Max}}\left\{\mathrm{S}_{\tau}\left(\mathrm{k}-\mathcal{M}_{0}((\tau, \mathrm{t}))\right)+\mathrm{X}_{\tau}\left(\mathrm{k}-\mathcal{M}_{0}((\tau, \mathrm{t}))\right)\right\}
$$

We assume that for each transition $t$, the sequence of its firing times $\left\{X_{t}(k)\right\}_{k=1}^{\infty}$ is a sequence of independent identically distributed (i.i.d.) integrable random variables and that $\left\{\mathrm{X}_{\mathrm{t}}(\mathrm{k})\right\}_{\mathrm{k}=1}^{\infty}$ for all $\mathrm{t} \in \mathrm{T}$ are mutually independent sequences.

It was proven in [1] that, under the foregoing assumptions, there exists a positive constant $\pi\left(\mathrm{M}_{0}\right)$ such that:

$$
\lim _{k \rightarrow \infty} S_{t}(k) / k=\lim _{k \rightarrow \infty} E\left[S_{t}(k)\right] / k=\pi\left(M_{0}\right) \text {, a.s. } \forall t \in T
$$

$\pi\left(M_{0}\right)$ is the average cycle time of the event graph.

Since $\left\{X_{t}(k)\right\}_{k=1}^{\infty}$ are sequences of i.i.d. random variables, the index $k$ is often omitted and we use $X_{t}$ to denote the firing time of transition $t$ whenever $k$ is not necessary. We further assume that the first and second moments of $X_{t}$ exist and denote by $m_{t}$ its mean value and by $\sigma_{t}$ its standard deviation, i.e. $m_{t}=E\left[X_{t}\right]$ and $\sigma_{t}^{2}=E\left[\left(X_{t}-m_{t}\right)^{2}\right]$. Note that the existence of the second moments implies that the random variables are integrable. 
Since any stochastic timed event graph is completely characterized by its net structure, its initial marking and the set of transition firing time sequences, it can be denoted by the triplet $\left(N, M_{0},\left\{X_{t}(k)\right\}\right)$.

Finally, the marking optimization problem can be defined as follows. Given a positive real value $C$, the marking optimization problem consists of finding an initial marking $\mathrm{M}_{0} \in \mathrm{IN}^{|\mathrm{P}|}$ in order to

minimize $\mathrm{f}_{\mathrm{U}}\left(\mathrm{M}_{0}\right)=\mathrm{U}^{\mathrm{T}} \cdot \mathrm{M}_{0}$

subject to the following constraint :

$\pi\left(\mathrm{M}_{0}\right) \leq \mathrm{C}$

where $U=\left(u_{1}, u_{2}, \ldots, u_{|P|}\right)^{T} \in\left(I^{+}\right)|P|$ is a $p$-invariant (also called P-semi flow).

In the marking optimization problem, the value $1 / \mathrm{C}$ can be considered as the minimal required throughput rate or productivity value. The vector $U$ can be considered as the unit cost of the resources (or tokens). The marking optimization problem consists of reaching the required performance while minimizing the total resource cost $\mathrm{U}^{\mathrm{T}} . \mathrm{M}_{0}$.

We remember that a p-invariant is a vector which associates to each place a nonnegative weight such that the total weighted sum of the tokens in the Petri net remains invariant whatever the transition firings. In the event graph case, each elementary circuit corresponds to a p-invariant.

The use of the p-invariant criterion should not be surprising. As a matter of fact, the number of tokens in each elementary circuit of the event graph indicates the number of resources of the related type. Hence, two initial markings with identical circuit counts give the same total resource cost. This implies that the cost function is a pinvariant criterion.

\section{EXAMPLES}

The purpose of this section is two-fold. First, we introduce an illustrative example to be used later. We then present some applications of the marking optimization in the field of manufacturing systems.

\subsection{An illustrative example}

We present here an example which later will be used to illustrate various points. The strongly connected event graph is presented in figure 1 (the self loop places are not given for the sake of simplicity). 


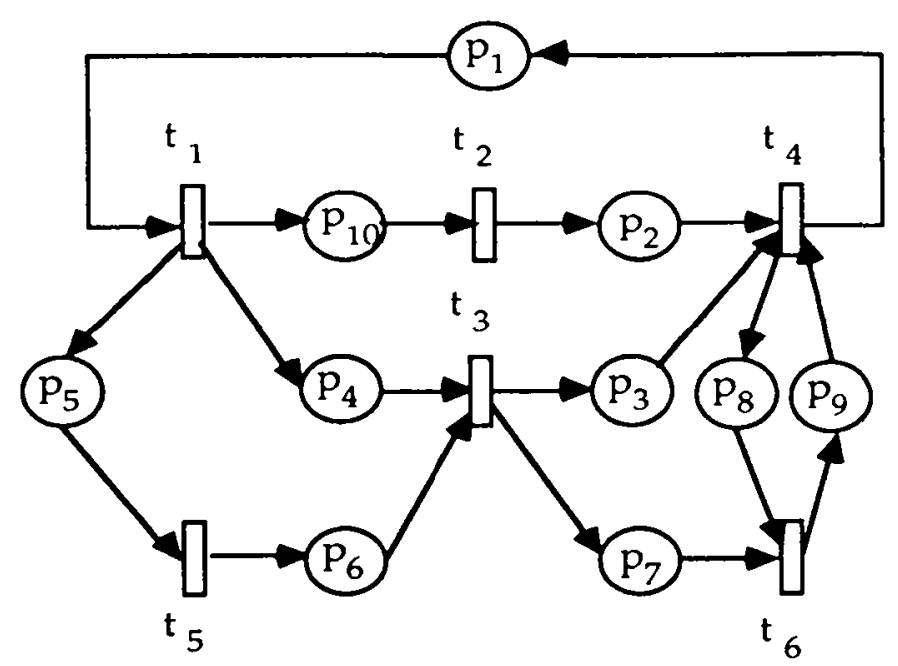

Fig. 1: A strongly connected event graph

The random variables $X_{1}, X_{2}, X_{3}, X_{4}, X_{5}, X_{6}$ are assigned to the transitions $t_{1}, t_{2}, t_{3}, t_{4}, t_{5}$ and $t_{6}$ respectively. Their probability density functions are as follows:

$$
\begin{aligned}
& X_{1}: f_{1}(x)=\left\{\begin{array}{l}
1 / 10 \text { if } x \in[0,10] \\
0 \text { otherwise }
\end{array}\right. \\
& X_{2}: f_{2}(x)=\left\{\begin{array}{l}
\left(1 / \beta^{\alpha} \Gamma(\alpha)\right) \cdot x^{\alpha-1} \exp (-x / \beta) \text { if } x \geq 0 \\
0 \text { otherwise }
\end{array}\right. \\
& \quad \text { with } \alpha=2 \text { and } \beta=5
\end{aligned}
$$

The marking optimization problem to be solved consists of:

$$
\begin{aligned}
& \text { minimizing } f_{U}\left(M_{0}\right)=U^{T} \cdot M_{0} \\
& \text { subject to the following constraint : } \\
& \pi\left(M_{0}\right) \leq C
\end{aligned}
$$

where $U=(3,1,1,1,1,1,1,1,2,1)^{\mathrm{T}}$.

\subsection{Modeling and dimensioning of closed production lines}

Consider a closed production line composed of a series of $m$ machines $\left(\mathcal{M}_{1}, \mathcal{M}_{2}, \ldots, \mathcal{M}_{\mathrm{m}}\right)$ separated by $m$ buffers $\left(B_{1}, B_{2}, \ldots, B_{m}\right)$. The in-process parts are carried on transportation resources such as pallets or carts. Assume that there are $K$ transportation resources. The buffer capacities are limited and are respectively $\mathrm{H}_{1}, \mathrm{H}_{2}$, $\ldots, H_{m}$. The processing times are generated by a set of random processes $\left\{X_{i}(k)\right\}$ where 
$\mathrm{X}_{\mathrm{i}}(\mathrm{k})$ denotes the processing time of the $\mathrm{k}$-th part on machine $\mathcal{M}_{\mathrm{i}}$. Figure 2 illustrates a three machine closed production line.

The behaviour of the system can be described as follows. When a pallet departs from the last machine, the part loaded on it is unloaded and a new part is loaded on it from an input buffer of the system. It then moves to buffer $B_{1}$ waiting the service of machine $\mathcal{M}_{1}$. After having been processed by $\mathcal{M}_{1}$, the pallet moves to buffer $B_{2}$ if it is not full. Otherwise, the pallet stays on machine $\mathcal{M}_{1}$ and the machine is blocked. This blocking situation persists until a part has been removed from the buffer $B_{2}$. The pallet then moves to buffer $B_{2}$. It continues in this manner through all the machines. Upon its departure from the last machine, the actual part is unloaded and a new part is loaded on the pallet. The process continues indefinitely.

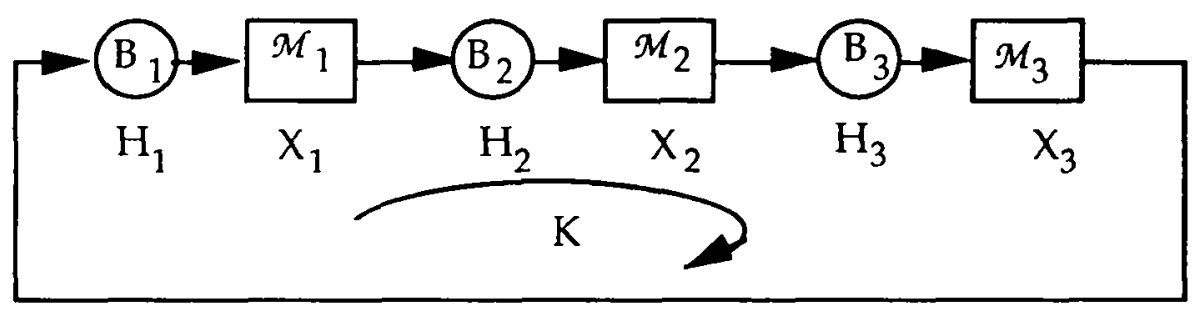

Figure 2 : A three machine closed production line

The design problem that we are interested in consists of determining the buffer capacities and the number of transportation resources in order to obtain a given average cycle time (or productivity) while

$$
\text { Minimizing } \sum_{\mathrm{i}=1}^{\mathrm{m}} \alpha_{\mathrm{i}} \mathrm{H}_{\mathrm{i}}+\beta \mathrm{K}
$$

where $\alpha_{i}$ is the unit cost related to the buffer capacity of $B_{i}$ and $\beta$ is the unit cost related to the transportation resources.

The Petri net model of the three machine closed production line is illustrated in Figure 3 and the Petri net model of the general case is similar.

The model can be decomposed into $m$ stages and each stage corresponds to a buffer and its downstream machines. For stage $i$, there are five places $F_{i}, I_{i}, Q_{i}, O_{i}$ and $W_{i}$ and four transitions: $T_{i}, L_{i}, t_{i}$ and $T_{i+1}$. The places $I_{i}, O_{i}$ and $W_{i}$ contain exactly one token collectively, and the position of the token indicates the state of the machine $\mathcal{M}_{\mathbf{i}}$. The machine is free (or processing parts, or blocked) if the token is in place $\mathrm{I}_{\mathrm{i}}$ (or $\mathrm{O}_{\mathrm{i}}$, or $\mathrm{W}_{\mathrm{i}}$ ). The number of tokens in place $F_{i}$ indicates the number of available storage places in stage $i$, and the number of tokens in place $Q_{i}$ indicates the number of pallets in buffer $\mathrm{B}_{\mathrm{i}}$. 


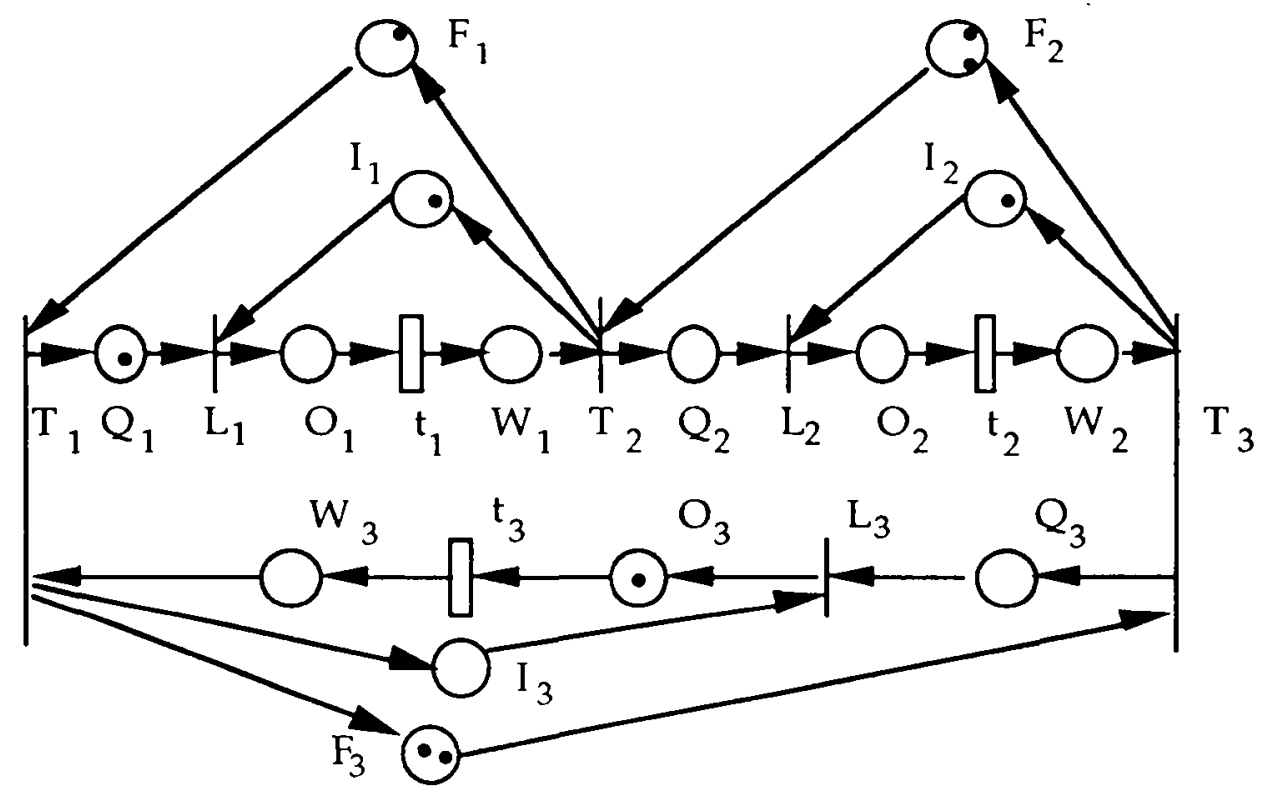

Figure 3 : The Petri net model of a 3-machine closed production line

The transition $T_{i}$ represents the transfer of a pallet from stage $i-1$ to stage $i . L_{i}$ corresponds to the loading operation of a pallet on the machine $\mathcal{M}_{\mathbf{i}} \cdot \mathbf{t}_{\mathbf{i}}$ models the operations performed by $\mathcal{M}_{\mathrm{i}}$. Only the transitions $t_{\mathrm{i}}$ are timed and the others are immediate transitions, i.e.

$$
\begin{array}{ll}
\mathrm{X}_{\mathrm{L}_{\mathrm{i}}}(\mathrm{k})=\mathrm{X}_{\mathrm{T}_{\mathrm{i}}}(\mathrm{k})=0 & \forall \mathrm{i}=1,2, \ldots, \mathrm{K} \text { and } \forall \mathrm{k} \\
\mathrm{X}_{\mathrm{t}_{\mathrm{i}}}(\mathrm{k})=\mathrm{X}_{\mathrm{i}}(\mathrm{k}) & \forall \mathrm{i}=1,2, \ldots, \mathrm{K} \text { and } \forall \mathrm{k}
\end{array}
$$

Finally, the Petri net model is a strongly connected event graph. There are $2 m+2$ elementary circuits. The elementary circuit $\left(\mathrm{I}_{\mathrm{i}} \mathrm{L}_{\mathrm{i}} \mathrm{O}_{\mathrm{i}} \mathrm{t}_{\mathrm{i}} \mathrm{W}_{\mathrm{i}} \mathrm{T}_{\mathrm{i}} \mathrm{I}_{\mathrm{i}}\right)$ represents the state evolution of machine $\mathcal{M}_{\mathrm{i}}$ and it contains exactly one token. The elementary circuit $\left(\mathrm{F}_{\mathrm{i}} \mathrm{T}_{\mathrm{i}} \mathrm{Q}_{\mathrm{i}} \mathrm{L}_{\mathrm{i}} \mathrm{O}_{\mathrm{i}} \mathrm{t}_{\mathrm{i}} \mathrm{W}_{\mathrm{i}} \mathrm{T}_{\mathrm{i}+1} \mathrm{~F}_{\mathrm{i}}\right)$ represents the storage space constraint of stage $\mathrm{i}$ and it contains exactly $\mathrm{H}_{\mathrm{i}}+1$ tokens. The elementary circuit $\left(\mathrm{T}_{1} \mathrm{Q}_{1} \mathrm{~L}_{1} \mathrm{O}_{1} \mathrm{t}_{1} \mathrm{~W}_{1} \mathrm{~T}_{2} \mathrm{Q}_{2} \mathrm{~L}_{2} \mathrm{O}_{2} \mathrm{t}_{2} \mathrm{~W}_{2} \ldots\right.$ $T_{m} Q_{m} L_{m} O_{m} t_{m} W_{m} T_{1}$ ) represents the distribution of the pallets and it contains exactly $K$ tokens. The elementary circuit $\left(\mathrm{T}_{1} \mathrm{~F}_{\mathrm{m}} \mathrm{T}_{\mathrm{m}} \mathrm{F}_{\mathrm{m}-1} \ldots \mathrm{T}_{2} \mathrm{~F}_{1} \mathrm{~T}_{1}\right)$ is a dual circuit of the last one and it contains exactly $\sum_{1 \leq i \leq m}\left(H_{i}+1\right)-K$ tokens.

The design problem corresponds to the marking optimization problem which consists of :

minimizing $\mathrm{f}_{\mathrm{U}}\left(\mathrm{M}_{0}\right)=\mathrm{U}^{\mathrm{T}} \cdot \mathrm{M}_{0}$ subject to the following constraint :

$\pi\left(\mathrm{M}_{0}\right) \leq \mathrm{C}$ 
where the p-invariant $U$ is defined as follows :

$$
\mathrm{U}_{\mathrm{p}}= \begin{cases}\alpha_{\mathrm{i}}, & \text { if } \mathrm{p}=\mathrm{F}_{\mathrm{i}} ; \\ \mathrm{G}, & \text { if } \mathrm{p}=\mathrm{I}_{\mathrm{i}} ; \\ \alpha_{\mathrm{i}}+\beta, & \text { if } \mathrm{p}=\mathrm{Q}_{\mathrm{i}} ; \\ \alpha_{\mathrm{i}}+\beta+\mathrm{G}, & \text { if } \mathrm{p}=\mathrm{O}_{\mathrm{i}} \text { or } \mathrm{p}=\mathrm{W}_{\mathrm{i}}\end{cases}
$$

In this formulation, a large number $G$ is introduced to guarantee that in the optimal solution, there is exactly one token in each elementary circuit related to the state of the machines.

\subsection{Dimensioning of manufacturing systems}

Event graph models have been proposed for more general manufacturing systems. They are repetitive manufacturing systems and include : transfer lines ([26]), job-shops $([9,12])$, Kanban systems $([12,26])$, and assembly/disassembly systems $([11,26])$.

In the case of transfer lines, the marking optimization of the Petri net model corresponds to the optimal determination of buffer capacities. In the case of job-shops, it corresponds to the minimization of the number of transportation resources. In the case of Kanban systems, it corresponds to the optimal determination of the kanban distribution. In the case of assembly/disassembly systems, it corresponds to the optimal determination of the buffer capacities and/or the number of transportation resources.

\section{PROPERTIES}

This section is devoted to the properties of the optimal solutions of the stochastic marking optimization problem. We first present some basic properties. Tight upper bounds and lower bounds of the average cycle times which allow to derive properties of the optimal markings are then presented. Finally, we establish the reachability conditions of a given cycle time $\mathrm{C}$.

\subsection{Some basic properties}

Property 1. ([6])

An initial marking $M_{0}$ is a live marking iff each elementary circuit contains at least one token, i.e. $\mathrm{M}_{0}(\gamma) \geq 1, \forall \gamma \in \Gamma$.

\section{Property 2.}

Assume that $M$ and $M_{0}$ are two markings with identical circuit counts, i.e. $M(\gamma)=$ $\mathrm{M}_{0}(\gamma), \forall \gamma \in \Gamma$. In this case, it holds that :

(a) $M_{0}$ and $M$ are mutually reachable; 
(b) $\pi(\mathrm{M})=\pi\left(\mathrm{M}_{0}\right)$;

(c) $f_{U}(M)=f_{U}\left(M_{0}\right)$.

In this property, the claim (a) was proved in [6], the claim (b) was proved in [25] while the claim (c) is obvious since $f_{U}(M)=U^{T} . M, f_{U}\left(M_{0}\right)=U^{T} . M_{0}$ and $U$ is a p-invariant.

Property 3.

Let $M_{1}$ and $M_{2}$ be two initial markings such that $M_{1}(\gamma) \leq M_{2}(\gamma), \forall \gamma \in \Gamma$. Then,

(a) $\exists M \in R\left(M_{1}\right)$ such that $M \leq M_{2}$, i.e. $M(p) \leq M_{2}(p), \forall p \in P$.

(b) $\pi\left(\mathrm{M}_{1}\right) \geq \pi\left(\mathrm{M}_{2}\right)$;

(c) $f_{U}\left(M_{1}\right) \leq f_{U}\left(M_{2}\right)$.

Proof :

The claim (a) is Theorem 10 in [6]. From Property 2, we have :

$\pi\left(M_{1}\right)=\pi(M)$ and $f_{U}\left(M_{1}\right)=f_{U}(M)$.

The monotonicity property with respect to the marking in [3] then implies that $\pi\left(\mathrm{M}_{2}\right)$ $\leq \pi(M)=\pi\left(M_{1}\right)$. Since $U$ is a vector of non-negative numbers, equation (2) implies that $f_{U}\left(M_{2}\right)=U^{T} \cdot M_{2} \geq U^{T} M=f_{U}(M)=f_{U}\left(M_{1}\right)$.

Q.E.D.

In the following, we need some stochastic ordering relations introduced by Stoyan [23]. In particular, the convex ordering relation and the strong ordering relation will be used. Let $\leq_{i c x}$ denote the convex ordering relation and $\leq_{s t}$ the strong ordering. Two random variables $X$ and $Y$ are said to satisfy the strong ordering relation (resp. the convex ordering relation), i.e. $X \leq_{s t} Y\left(\right.$ resp. $X \leq_{i c x} Y$ ) if the following inequality

$$
\mathrm{E}[\mathrm{g}(\mathrm{X})] \leq \mathrm{E}[\mathrm{g}(\mathrm{Y})]
$$

holds for all monotone nondecreasing (resp. convex monotone nondecreasing) function $g: I R \rightarrow I R$ provided that the expectations exist.

Property 4.

Consider two stochastic timed event graphs with the same net structure STEG1 $=(\mathrm{N}$,

-,$\left.\left\{X_{t}(k)\right\}\right)$ and STEG2 $=\left(N, \bullet,\left\{Y_{t}(k)\right\}\right)$ and let $M_{1}$ (resp. $\left.M_{2}\right)$ be the optimal solution for STEG1 (resp. STEG2). If

$$
X_{t} s_{i c x} Y_{t}, \quad \forall t \in T,
$$

then

$$
f_{U}\left(M_{1}\right) \leq f_{U}\left(M_{2}\right)
$$

Since $\leq_{\mathrm{icx}}$ ordering implies the strong ordering $\leq_{\mathrm{st}}$, the property still holds if the $\leq_{\mathrm{icx}}$ ordering is replaced by the $\leq_{s t}$ ordering. 
Proof :

Let $\pi^{1}\left(M_{0}\right)$ (resp. $\pi^{2}\left(M_{0}\right)$ ) be the average cycle time of STEG1 (resp. STEG2) when the initial marking is $M_{0}$. From the definition of $M_{1}$ and $M_{2}$, we have :

$$
\pi^{1}\left(\mathrm{M}_{1}\right) \leq \mathrm{C} \text { and } \pi^{2}\left(\mathrm{M}_{2}\right) \leq \mathrm{C} .
$$

Let us prove the property by contradiction and assume that $f_{U}\left(M_{1}\right)>f_{U}\left(M_{2}\right)$. From the monotonicity property of the average cycle time with respect to the transition firing times in [3],

$$
\pi^{1}\left(\mathrm{M}_{2}\right) \leq \pi^{2}\left(\mathrm{M}_{2}\right) \leq \mathrm{C}
$$

which implies that $M_{2}$ is also a solution for STEG1 and that $M_{1}$ cannot be the optimal solution for STEG1. This is in contradiction with our assumption.

Q.E.D.

Property 2 claims that if $\mathrm{M}_{0}$ is an optimal solution to the marking optimization problem, then any marking reachable from $\mathrm{M}_{0}$ is also an optimal solution. Property 3 claims that the average cycle time is non-increasing with regard to the initial marking while the p-invariant criterion is non-decreasing. Property 4 claims that the optimum criterion value is non-decreasing in transition firing times under stochastic comparison relations.

\subsection{Lower bounds of the average cycle time and the p-invariant criterion}

Given the initial marking and the net structure, it was proven in $[3,4]$ that the minimal average cycle time is obtained in the deterministic case as indicated in the following property.

Property $5([3,4])$.

$$
\pi\left(\mathrm{M}_{0}\right) \geq \pi^{\mathrm{D}}\left(\mathrm{M}_{0}\right)
$$

where $\pi^{D}\left(M_{0}\right)$ denotes the average cycle time of the deterministic timed event graph $\left(N, M_{0},\left\{Y_{t}(k)\right\}\right)$ with $Y_{t}(k)=m_{t}$, i.e.

$$
\pi^{\mathrm{D}}\left(\mathrm{M}_{0}\right)=\underset{\gamma \in \Gamma}{\operatorname{Max}} \frac{\sum_{\mathrm{t} \in \gamma} \mathrm{m}_{\mathrm{t}}}{\mathrm{M}_{0}(\gamma)}
$$

From this property, the following corollary can be shown. It shows that the pinvariant criterion reaches its minimum when the firing times become deterministic.

Corollary 1 .

$$
f_{U}\left(M^{S}\right) \geq f_{U}\left(M^{D}\right)
$$


where $M^{S}$ (resp. $M^{D}$ ) denotes the optimal solution of the marking optimization problem of the stochastic timed event graph $\left(N, M_{0},\left(X_{t}(k)\right)\right.$ ) (resp. the deterministic event graph (N, $\left.\left.M_{0},\left(m_{t}\right\}\right)\right)$.

Remark that this corollary can also be derived from Property 4 by considering the relation $X_{t} s_{i c x} E\left[X_{t}\right]=m_{t}$.

\subsection{Upper bounds and properties of optimal solutions}

The superposition properties in [25] are particularly useful in deriving upper bounds of the average cycle time. In particular, the main superposition property claims that the average cycle time is sub-additive when the transition firing times are generated by the superposition of two sets of sequences of random variables.

\section{Main superposition property.}

Assuming that $x_{t}=x_{t}^{1}+x_{t}^{2}, \forall t \in T$ where $x_{t}^{1}$ and $x_{t}^{2}$ are non-negative random variables, let $\pi^{1}\left(\mathrm{M}_{0}\right)$ (resp. $\left.\pi^{2}\left(\mathrm{M}_{0}\right)\right)$ be the average cycle time of the stochastic timed event graph $\left(N, M_{0},\left\{X_{t}^{1}(k)\right\}\right)$ (resp. $\left(N, M_{0},\left\{X_{t}^{2}(k)\right\}\right)$ ). Then it holds that :

$$
\pi\left(\mathrm{M}_{0}\right) \leq \pi^{1}\left(\mathrm{M}_{0}\right)+\pi^{2}\left(\mathrm{M}_{0}\right)
$$

Using this property, the following upper bound can be easily derived.

Property 6.

$$
\pi\left(\mathrm{M}_{0}\right) \leq \inf _{\mathrm{Z} \geq 0}\left\{\operatorname{Max}_{\gamma \in \Gamma} \frac{\sum_{\mathrm{t} \in \gamma} \mathrm{z}_{\mathrm{t}}}{\mathrm{M}_{0}(\gamma)}+\pi^{2}\left(\mathrm{M}_{0}, \mathrm{Z}\right)\right\}
$$

where $Z$ denotes the vector of constants $\left(z_{1}, z_{2}, \ldots, z_{|T|}\right)^{T}$ and $\pi^{2}\left(M_{0}, Z\right)$ denotes the average cycle time of the stochastic timed event graph $\left(N, M_{0},\left\{X_{t}^{2}(k)\right\}\right)$ with $X_{t}^{2}(k)=\left(X_{t}(k)-z_{t}\right)^{+}$.

Combining this upper bound and the upper bound presented in [4], the following property can be shown.

\section{Property 7.}

$$
\pi\left(\mathrm{M}_{0}\right) \leq \pi^{\mathrm{D}}\left(\mathrm{M}_{0}\right)+\operatorname{Inf}_{\mathrm{Z} \in \mathcal{E}}\left\{\sum_{t \in \mathrm{T}} \mathrm{E}\left[\left(\mathrm{X}_{\mathrm{t}}-\mathrm{z}_{\mathrm{t}}\right)^{+}\right]\right\}
$$

where

$$
\mathcal{E}=\left\{\mathrm{z} / \mathrm{z}_{\mathrm{t}} \geq \mathrm{m}_{\mathrm{t}}, \forall \mathrm{t} \in \mathrm{T} \text { and } \sum_{t \in \gamma} \mathrm{z}_{\mathrm{t}} \leq \pi^{\mathrm{D}}\left(\mathrm{M}_{0}\right) \mathrm{M}(\gamma), \forall \gamma \in \Gamma\right\}
$$


This bound shows that the firing time randomness of transitions belonging to noncritical elementary circuits has little effect on the average cycle time of the whole system. The effect of their randomness can almost be completely canceled by taking large values for $z_{t}$.

By taking $z_{t}=m_{t}, \forall t \in T$ and by using arguments similar to those in [25], the following upper bound can be derived.

\section{Corollary 2.}

$$
\pi\left(\mathrm{M}_{0}\right) \leq \pi^{\mathrm{D}}\left(\mathrm{M}_{0}\right)+\sum_{\mathrm{t} \in \mathrm{T}} \sigma_{\mathrm{t}}
$$

From these bounds, the following corollary can be easily shown.

\section{Corollary 3.}

Let $\mathrm{M}^{\mathrm{S}}$ and $\mathrm{M}^{\mathrm{D}}$ be the markings defined in corollary 1 . If the following relation holds

$$
\operatorname{Inf}_{Z \in \mathcal{E}}\left\{\sum_{t \in T} E\left[\left(X_{t}-z_{t}\right)^{+}\right]\right\} \leq C-\pi^{D}\left(M^{D}\right)
$$

then

$$
M^{S}=M^{D}
$$

Recall that $C$ is the average cycle time to be reached. This corollary provides a sufficient condition under which the optimal solution for the deterministic timing case remains optimal.

We also consider a special case in which the initial marking is a multiple of another live marking. By using the main superposition property, the following tighter upper bound can be derived (see [20]).

Property 8.

$$
\pi\left(\mathrm{nM}_{0}\right) \leq \pi^{\mathrm{D}}\left(\mathrm{M}_{0}\right)+\inf _{\mathrm{Z} \in \mathcal{E}}\left\{\sum_{\mathrm{t} \in \mathrm{T}} \mathrm{E}\left[\left(\frac{1}{\mathrm{n}} \sum_{\mathrm{k}=1}^{\mathrm{n}} \mathrm{X}_{\mathrm{t}}(\mathrm{k})-\mathrm{z}_{\mathrm{t}}\right)^{+}\right]\right\}
$$

where $\mathcal{E}$ is the set of vectors defined in Property 6.

From this property, we can prove the following corollary which shows that multiplying the number of tokens can reduce the variance of the transition firing times.

Corollary 4.

$$
\pi\left(\mathrm{nM}_{0}\right) \leq \pi^{\mathrm{D}}\left(\mathrm{M}_{0}\right)+\sum_{\mathrm{t} \in \mathrm{T}} \frac{\sigma_{\mathrm{t}}}{\sqrt{\mathrm{n}}}
$$




\subsection{Cycle time reachability}

This subsection is devoted to the reachability of a given cycle time C. In the following, we prove that any cycle time $\mathrm{C}$ which is strictly greater than the maximal average transition firing time can be reached, while any $C$ which is strictly smaller than the maximal transition firing time cannot be reached. Necessary and sufficient condition for the reachability of a given cycle time $C$ equal to the maximal average transition firing time is also given.

Let $\mathrm{C}^{*}$ be the maximal average transition firing time, i.e.

$$
C^{*}=\underset{t \in T}{\operatorname{Max}}\left\{m_{t}\right\}
$$

Property 9.

$$
\pi\left(\mathrm{M}_{0}\right) \geq \mathrm{C}^{*}, \forall \mathrm{M}_{0}
$$

Proof :

From Property 5,

$$
\pi\left(\mathrm{M}_{0}\right) \geq \pi^{\mathrm{D}}\left(\mathrm{M}_{0}\right)
$$

Combining with Property 3 in [12], we obtain :

$$
\pi\left(\mathrm{M}_{0}\right) \geq \pi^{\mathrm{D}}\left(\mathrm{M}_{0}\right) \geq \mathrm{C}^{*}
$$

Property 10.

Let $\mathrm{M}_{0}=\mathrm{n} 1_{|\mathrm{P}|}$ where $1_{|\mathrm{P}|}$ is a vector with all its components equal to 1 . Then,

$$
\pi\left(\mathrm{M}_{0}\right) \leq \mathrm{C}^{*}+\sum_{\mathrm{t} \in \mathrm{T}} \frac{\sigma_{\mathrm{t}}}{\sqrt{\mathrm{n}}}
$$

Proof :

From Corollary 4,

$$
\pi\left(\mathrm{M}_{0}\right) \leq \pi^{\mathrm{D}}\left(1_{|\mathrm{P}|}\right)+\sum_{\mathrm{t} \in \mathrm{T}} \frac{\sigma_{\mathrm{t}}}{\sqrt{\mathrm{n}}}=\mathrm{C}^{*}+\sum_{\mathrm{t} \in \mathrm{T}} \frac{\sigma_{\mathrm{t}}}{\sqrt{\mathrm{n}}}
$$

Q.E.D.

Recall that similar property has also been shown in [15] by means of a so called N-POM constrained operating mode.

The following property presents the necessary and sufficient condition for the reachability of $C^{*}$ proposed in $[14,19]$. 
Property 11 .

$C^{*}$ is reachable iff there exists $t^{*} \in T$ such that

$$
\mathrm{P}\left[\mathrm{X}_{\mathrm{t}^{*}}=\underset{\mathrm{t} \in \mathrm{T}}{\operatorname{Max}} \mathrm{X}_{\mathrm{t}}\right]=1
$$

Furthermore,

(a) If this condition holds, $\pi\left(n 1_{|P|}\right)=C^{*}, \forall n \geq 1$.

(b) If it does not hold, $\pi\left(\mathrm{M}_{0}\right)>\mathrm{C}^{*}, \forall \mathrm{M}_{0}$

\section{A HEURISTIC SOLUTION}

In section 4 , we proved that it is always possible to reach a mean cycle time smaller than a given value $C$ with a finite number of tokens, provided $C>C^{*}$. The purpose of this section is to present a heuristic solution to the marking optimization problem introduced in Section 2.

\subsection{A heuristic solution to the marking optimization problem}

The heuristic algorithm presented hereafter leads to a near-optimal solution to the marking optimization problem. It is a two-phases algorithm. Its first phase consists of computing the optimal solution to the deterministic problem obtained by assigning to each transition the mean value of the related random variable. We use the algorithm presented in [12] to solve this problem.

The second phase of the algorithm is an iterative process. In each iteration, a new token is added in a place to be determined as long as the given cycle time $C$ is not obtained.

In order to determine the most adequate place to which the new token is added, we first evaluate by simulation the average cycle time $\pi\left(\mathrm{M}_{0}\right)$. If it is smaller than $C$, the given cycle time $C$ has been obtained and we keep the marking $M_{0}$ as the optimal solution. Otherwise, we evaluate the sensitivity of the average cycle time with respect to the marking of place $\mathrm{p} \Delta \pi\left(\mathrm{M}_{0}, \mathrm{p}\right)$ defined as follows :

$$
\Delta \pi\left(\mathrm{M}_{0}, \mathrm{p}\right)=\pi\left(\mathrm{M}_{0}\right)-\pi^{\circ}\left(\mathrm{M}_{0}, \mathrm{p}\right)
$$

where $\pi^{\circ}\left(\mathrm{M}_{0}, \mathrm{p}\right)$ is the average cycle time of the event graph when an extra token is added in place $p$, i.e. $\pi^{\circ}\left(M_{0}, p\right)=\pi\left(M_{1}\right)$ with $M_{1}(p)=M_{0}(p)+1$ and $M_{1}(s)=M_{0}(s), \forall s \in P$ and $s \neq p$.

The basic idea of this heuristic procedure is to add a token in a place such that the sensitivity is as large as possible while the criterion value $f_{U}\left(M_{0}\right)$ increases as little as possible. To achieve this, we choose a place $\mathrm{p}^{*}$ which 
maximizes $h_{U}\left(M_{0}, p\right)=\Delta \pi\left(M_{0}, p\right) / u_{p}$

where $u_{1}, u_{2}, \ldots, u_{|P|}$ are coefficients of the p-invariant criterion.

The details of this heuristic algorithm is given in the following :

\section{ALGORITHM 1.}

First phase: Computation of an initial solution

1. Solve the marking optimization problem of the deterministic timed event graph $\left(N, \bullet,\left\{m_{t}\right\}\right)$ and let the optimal initial marking be $M^{D}$

Recall that $m_{t}$ is the mean firing time of transition $t$, i.e. $m_{t}=E\left[X_{t}(k)\right]$. We use the algorithms presented in [12] to solve this problem. The optimal solution $M^{D}$ is specified as the number of tokens in each place at the initial state.

2. Set $\mathrm{M}_{0}=\mathrm{M}^{\mathrm{D}}$

Second phase: Increase adequately the set of tokens

3. Simulate the stochastic timed event graph $\left(N, M_{0},\left\{X_{t}(k)\right\}\right)$ in order to obtain the average cycle time $\pi\left(\mathrm{M}_{0}\right)$ by using Algorithm 0 .

4. If $\pi\left(\mathrm{M}_{0}\right)<\mathrm{C}$, stop the computation.

5. Evaluate the sensitivities of the average cycle time with respect to the marking of the places $\Delta \pi\left(M_{0}, p\right)$.

6. Compute the place $\mathrm{p}^{*}$ which maximizes $h_{U}\left(M_{0}, p\right)=\Delta \pi\left(M_{0}, p\right) / U_{p}$.

7. Add a token to place $p^{*}$, i.e. $M_{0}\left(p^{*}\right):=M_{0}\left(p^{*}\right)+1$ and go to step 3 .

As can be noticed, IPI extra simulations are needed in each iteration in order to evaluate the sensitivities of the average cycle time with respect to the marking of the places, i.e. $\Delta \pi\left(\mathrm{M}_{0}, \mathrm{p}\right)$. In the next subsection, we propose an approximate evaluation of these sensitivities which avoids the extra simulations.

Let us consider now the simulation of a stochastic timed event graph. Thanks to the ergodicity relation (2), we can use the evolution equation (1) to simulate a stochastic timed event graph instead of the classical discrete event simulation technique. This approach turns out to be more efficient than the discrete simulation technique.

ALGORITHM 0 (Simulation of a stochastic timed event graph)

1. Choose a simulation cycle $\mathrm{K}$.

2. Compute a sequence of transitions $\left(\sigma_{1}, \ldots, \sigma_{|\mathrm{T}|}\right)$ firable from the initial marking $\mathbf{M}_{0}$ in which each transition appears exactly once.

3. Set $S_{t}(k):=0$, for all $t \in T$ and for all $k \leq 0$.

4. For $\mathrm{k}=1$ to $\mathrm{K}$ do

4.1. Generate the random variables $X_{t}(k)$ for all $t \in T$ 
4.2. For $\mathrm{t}=\sigma_{1}$ to $\sigma_{|\mathrm{T}|}$ do

$$
\mathrm{S}_{\mathrm{t}}(\mathrm{k})=\operatorname{Max}_{\tau \in \operatorname{in}(\mathrm{t})}\left\{\mathrm{S}_{\tau}\left(\mathrm{k}-\mathcal{M}_{0}((\tau, \mathrm{t}))\right)+\mathrm{X}_{\tau}\left(\mathrm{k}-\mathcal{M}_{0}((\tau, \mathrm{t}))\right)\right\}
$$

5. $\pi\left(\mathrm{M}_{0}\right)=\mathrm{S}_{\sigma 1}(\mathrm{~K}) / \mathrm{K}$

The existence of the sequence $\left(\sigma_{1}, \ldots, \sigma_{|\mathrm{T}|}\right)$ was proved in [6]. Since $\left(\sigma_{1}, \ldots, \sigma_{|\mathrm{T}|}\right)$ is firable from the initial marking $M_{0}$, the $k$-th firing of any transition $t=\sigma_{i}$ is independent of the k-th firings of the transitions $\sigma_{i+1}, \ldots, \sigma_{|\mathrm{T}|}$. This guarantees the computability of $\mathrm{S}_{\mathrm{t}}(\mathrm{k})$ in step 4.2 .

\subsection{Approximate evaluation of $\Delta \pi\left(\mathbf{M}_{0}, \mathrm{p}\right)$}

The basic idea of this approximate evaluation is to make use of additional information which can be obtained from the simulation of step 3 of the algorithm 1 .

For this purpose, let us consider the evolution equation (1) that we use to simulate the system. Rewrite this equation as follows:

$$
\mathrm{S}_{\mathrm{t}}(\mathrm{k})=\underset{\tau \in \operatorname{in}(\mathrm{t})}{\operatorname{Max}}\left\{\mathrm{S}_{\tau}\left(\mathrm{k}-\mathcal{M}_{0}((\tau, \mathrm{t}))\right)+\mathrm{X}_{\tau}\left(\mathrm{k}-\mathcal{M}_{0}((\tau, \mathrm{t}))\right)\right\}
$$

For transition $t$, let $\tau(t, k) \in$ in $(t)$ be the transition which triggers the $k$-th firing of transition $t$ and let $\rho(t, k)$ denote the place connecting transition $\tau(t, k)$ to $t$. Equation (10) can be rewritten as follows :

$$
\mathrm{S}_{\mathrm{t}}(\mathrm{k})=\mathrm{S}_{\tau(\mathrm{t}, \mathrm{k})}\left(\mathrm{k}-\mathscr{M}_{0}(\rho(\mathrm{t}, \mathrm{k}))\right)+\mathrm{X}_{\tau(\mathrm{t}, \mathrm{k})}\left(\mathrm{k}-\mathfrak{M}_{0}(\rho(\mathrm{t}, \mathrm{k}))\right)
$$

This equation implies that the transition $t$ waits for the arrival of a new token in place $\rho(t, k)$ to start its $k$-th firing.

We also compute the following quantities :

$$
\begin{aligned}
& \Pi(\mathrm{p}, \mathrm{k})=\frac{1}{\mathrm{k}} \sum_{\mathrm{i}=1}^{\mathrm{k}} 1\left(\mathrm{p}=\rho\left(\mathrm{p}^{\bullet}, \mathrm{i}\right)\right) \\
& \mathrm{S}_{\mathrm{t}}^{\circ}(\mathrm{k})=\underset{\tau \in \operatorname{in}(\mathrm{t}) \backslash\{\tau(\mathrm{t}, \mathrm{k})\}}{\operatorname{Max}}\left\{\mathrm{S}_{\tau}\left(\mathrm{k}-\mathcal{M}_{0}((\tau, \mathrm{t}))\right)+\mathrm{X}_{\tau}\left(\mathrm{k}-\mathcal{M}_{0}((\tau, \mathrm{t}))\right)\right\} \\
& \mathrm{W}(\mathrm{p}, \mathrm{k})=\frac{1}{\mathrm{k} \Pi(\mathrm{p}, \mathrm{k})} \sum_{\mathrm{i}=1}^{\mathrm{k}} 1\left(\mathrm{p}=\rho\left(\mathrm{p}^{\bullet}, \mathrm{i}\right)\right) *\left(\mathrm{~S}_{\mathrm{p}} \cdot(\mathrm{i})-\mathrm{S}_{\mathrm{p}}^{\circ} \cdot(\mathrm{i})\right)
\end{aligned}
$$

Clearly, $\Pi(p, \infty)$ is the steady-state probability that the downstream transition of place $p$ waits for the arrival of a new token in place $p$ to start a new firing. $S_{t}^{\circ}(k)$ is the instant 
of the k-th firing initiation of transition $t$ assuming that $\tau(t, k)$ is not critical for this $k$ th firing. $S_{t}(i)-S_{t}^{o}(i)$ is then the gain in the $i$-th firing initiation instant of transition $t$ assuming that $\tau(t, k)$ is not critical for this $k$-th firing. $W(p, \infty)$ is the average gain in the firing initiation instant of transition $\mathrm{p} \bullet$ related to place $\mathrm{p}$.

We notice that adding a token in place $p$ reduces the number of times that its downstream transition waits for the arrival of a new token to start a new firing.

From the above remarks, we conjecture that adding a token in place $p$ has almost the same effect as reducing the firing time of transition $p \bullet$ by $\Pi(p, \infty) * W(p, \infty)$, i.e.

$$
\pi^{\circ}\left(\mathrm{M}_{0}, \mathrm{p}\right) \approx \pi^{1}\left(\mathrm{M}_{0}, \mathrm{p}^{\bullet},-\Pi(\mathrm{p}, \infty) \mathrm{W}(\mathrm{p}, \infty)\right)
$$

where $\pi^{1}\left(M_{0}, \tau, \Delta\right)$ is the average cycle time of stochastic timed event graph $\left(N, M_{0}\right.$, $\left.\left\{Y_{t}(\tau, \Delta, k)\right\}\right)$ with $Y_{\tau}(\tau, \Delta, k)=X_{\tau}(k)+\Delta$ and $Y_{t}(\tau, \Delta, k)=X_{t}(k)$ for all $t \neq \tau$.

Consider the derivative of $\pi^{1}\left(M_{0}, \tau, \Delta\right)$ with respect to $\Delta$ that we denote as $\Delta \pi^{1}\left(M_{0}, \tau\right)$, i.e.

$$
\Delta \pi^{1}\left(\mathrm{M}_{0}, \tau\right)=\frac{\mathrm{d} \pi^{1}\left(\mathrm{M}_{0}, \tau, \Delta\right)}{\mathrm{d} \Delta}=\lim _{\Delta \rightarrow 0} \frac{\pi^{1}\left(\mathrm{M}_{0}, \tau, \Delta\right)-\pi\left(\mathrm{M}_{0}\right)}{\Delta}
$$

From relations $(8,15,16)$, we have

$$
\Delta \pi\left(\mathrm{M}_{0}, \mathrm{p}\right) \approx \Delta \pi^{1}\left(\mathrm{M}_{0}, \mathrm{p} \bullet\right) \Pi(\mathrm{p}, \infty) \mathrm{W}(\mathrm{p}, \infty)
$$

In this relation, $\Pi(p, \infty)$ and $W(p, \infty)$ can be obtained from the simulation of step 3 of the algorithm 1. In the next section, we show that the derivative can also be obtained from the same simulation by infinitesimal perturbation analysis (IPA) approach (see $[10,24]$ for surveys of the IPA technique).

\section{SENSITIVITY WITH RESPECT TO THE FIRING TIMES}

This section is devoted to the sensitivity analysis with respect to the firing times. We first present some general results. These results are then used to obtain the derivative needed in the heuristic algorithm proposed in Section 5.

\subsection{Some general results}

In order to perform perturbation analysis, let us consider a family of stochastic timed event graphs $\operatorname{STEG}(\theta)=\left(N, M_{0},\left\{X_{t}(k, \theta)\right\}\right) \cdot$ where $\theta$ is a real-valued parameter. The transition firing times are functions of $\theta$ and are defined as follows :

$$
X_{t}(k, \theta)=f_{t}\left(z_{t}(k), \theta\right)
$$


where $\mathrm{f}_{\mathrm{t}}(\bullet, \bullet): \mathrm{IR}^{+} \mathrm{xIR} \rightarrow \mathrm{IR}^{+}$and $z_{t}(\mathrm{k})$ for all $\mathrm{t}$ and $\mathrm{k}$ are random variables uniformly distributed on $[0,1]$.

For STEG( $\theta)$, the equations (10), (11) and (2) can be written as follows:

$$
\begin{aligned}
& \mathrm{S}_{\mathrm{t}}(\mathrm{k}, \theta)=\operatorname{Max}_{\tau \in \operatorname{in}(\mathrm{t})}\left\{\mathrm{S}_{\tau}\left(\mathrm{k}-\mathcal{M}_{0}((\tau, \mathrm{t})), \theta\right)+\mathrm{X}_{\tau}\left(\mathrm{k}-\mathcal{M}_{0}((\tau, \mathrm{t})), \theta\right)\right\} \\
& \mathrm{S}_{\mathrm{t}}(\mathrm{k}, \theta)=\mathrm{S}_{\tau(\mathrm{t}, \mathrm{k}, \theta)}\left(\mathrm{k}-\mathcal{M}_{0}(\rho(\mathrm{t}, \mathrm{k})), \theta\right)+\mathrm{X}_{\tau(\mathrm{t}, \mathrm{k}, \theta)}\left(\mathrm{k}-\mathcal{M}_{0}(\rho(\mathrm{t}, \mathrm{k}, \theta)), \theta\right)
\end{aligned}
$$

and

$$
\lim _{\mathrm{k} \rightarrow \infty} \mathrm{S}_{\mathrm{t}}(\mathrm{k}, \theta) / \mathrm{k}=\lim _{\mathrm{k} \rightarrow \infty} \mathrm{E}\left[\mathrm{S}_{\mathrm{t}}(\mathrm{k}, \theta)\right] / \mathrm{k}=\pi\left(\mathrm{M}_{0}, \theta\right), \text { a.s. } \forall \mathrm{t} \in \mathrm{T}
$$

where $S_{t}(k, \theta), \tau(t, k, \theta), \rho(t, k, \theta)$ and $\pi\left(M_{0}, \theta\right)$ are notations similar to $S_{t}(k), \tau(t, k), \rho(t, k)$ and $\pi\left(\mathrm{M}_{0}\right)$.

Consider the following regularity conditions :

(A1) Every $f_{t}\left(z_{t}(k), \theta\right)$ is, almost surely, a continuously differentiable function of $\theta$.

(A2) For every $\theta, t$ and $k, P\left(T_{\tau}(k, \theta)=T_{s}(k, \theta)\right.$, for some $s, \tau \in \operatorname{int}(t)$ and $\left.s \neq \tau\right)=0$ where

$$
\mathrm{T}_{\tau}(\mathrm{k}, \theta)=\mathrm{S}_{\tau}\left(\mathrm{k}-\mathcal{M}_{0}((\tau, \mathrm{t})), \theta\right)+\mathrm{X}_{\tau}\left(\mathrm{k}-\mathcal{M}_{0}((\tau, \mathrm{t})), \theta\right)
$$

(A3) For every $\theta, t$ and $k, \dot{f_{t}}\left(z_{t}(k), \theta\right) \geq 0$ and $E\left[\sup _{\theta} \dot{f}_{t}^{\prime}\left(z_{t}(k), \theta\right)\right]<\infty$ where

$$
f_{t}^{\prime}\left(z_{t}(k), \theta\right)=\frac{\partial \mathrm{f}_{t}\left(z_{t}(k), \theta\right)}{\partial \theta}
$$

Since the (CX) condition introduced in [7] holds in the evolution equation (19), the following properties hold thanks to Lemma 4.1., Theorem 4.1. and Theorem 4.2 in [7].

\section{Property 12.}

Suppose that (A1) and (A2) hold. Then for all $\theta, t$ and $k, S_{t}(k, \theta)$ is almost surely differentiable at $\theta$ and

$$
\frac{\partial \mathrm{S}_{\mathrm{t}}(\mathrm{k}, \theta)}{\partial \theta}=\mathrm{f}_{\tau(\mathrm{t}, \mathrm{k}, \theta)}^{\prime}\left(\mathrm{z}_{\tau(\mathrm{t}, \mathrm{k}, \theta)}(\mathrm{k}), \theta\right)+\frac{\partial \mathrm{S}_{\tau(\mathrm{t}, \mathrm{k}, \theta)}\left(\mathrm{k}-\mathcal{M}_{0}(\rho(\mathrm{t}, \mathrm{k}, \theta)), \theta\right)}{\partial \theta}
$$

Property 13 (Unbiasedness).

Suppose that (A1), (A2) and (A3) hold. Then for all $\theta, t$ and $k, E\left[S_{t}(k, \theta)\right]$ is differentiable and

$$
E\left[\frac{\partial S_{t}(k, \theta)}{\partial \theta}\right]=\frac{\partial E\left[S_{t}(k, \theta)\right]}{\partial \theta}
$$

\section{Property 14 (Strong consistency).}

Suppose that (A1), (A2) and (A3) hold. Assume further that the following limit

$$
\lim _{k \rightarrow \infty} \frac{\partial S_{t}(k, \theta)}{\partial \theta} / k
$$


exists almost surely. Then $\pi\left(\mathrm{M}_{0}, \theta\right)$ is differentiable for every $\theta$ and

$$
\frac{\partial \pi\left(\mathrm{M}_{0}, \theta\right)}{\partial \theta}=\lim _{\mathrm{k} \rightarrow \infty} \frac{\partial \mathrm{S}_{\mathrm{t}}(\mathrm{k}, \theta)}{\partial \theta} / \mathrm{k}, \text { a.s. } \forall \mathrm{t} \in \mathrm{T}
$$

General conditions for the existence of the limit (22) have not be obtained. Nevertheless, we can establish the following sufficient condition and we believe that this limit exists under fairly wide conditions.

Property 15 (Sufficient condition of strong consistency).

Suppose that (A1), (A2) and (A3) hold. Assume further that there exists at least one transition $t^{*} \in T$ whose firing times cannot be defined on a finite support, i.e. $P\left(X_{t^{*}}(k)\right.$ $\geq K\}>0$, for all $K>0$. Then $\pi\left(M_{0}, \theta\right)$ is differentiable for every $\theta$ and

$$
\frac{\partial \pi\left(\mathrm{M}_{0}, \theta\right)}{\partial \theta}=\lim _{\mathrm{k} \rightarrow \infty} \frac{\partial \mathrm{S}_{\mathrm{t}}(\mathrm{k}, \theta)}{\partial \theta} / \mathrm{k} \text {, a.s. } \forall \mathrm{t} \in \mathrm{T}
$$

Proof :

Thanks to Property 14, we only need to prove the existence of the limit (22). In the following we use regeneration to prove it.

Notice that if the transition $\mathrm{t}^{*}$ is frozen and the transitions are fired as soon as they are enabled, then the system stops at a unique marking $M^{*}$ (see [21]) after finite number of transition firings. This shows that there exists a positive value $\mathrm{K}^{*}>0$ such that whenever $X_{t^{*}}(k) \geq K^{*}$, the probability of reaching marking $M^{*}$ is non null whatever the initial marking.

Assuming that the initial marking $\mathrm{M}_{0}$ is the one obtained by firing once transition $\mathrm{t}^{*}$ at marking $\mathrm{M}^{*}$. Notice that this assumption can easily be relaxed. The instants at which the system leaves the marking $M^{*}$ are regeneration points. Since $P\left\{X_{t^{*}}(k) \geq K^{*}\right\}$ $>0$ for all $k$, the regenerative cycles have finite length and all the transitions fire exactly the same number of times in each cycle. From Theorem 3.6.1. in [18], we have:

$$
\lim _{\mathrm{k} \rightarrow \infty} \frac{\partial \mathrm{S}_{\mathrm{t}}(\mathrm{k}, \theta)}{\partial \theta} / \mathrm{k}=\frac{\mathrm{E}\left[\frac{\partial \mathrm{S}_{\mathrm{t}}(\mathrm{K} 1, \theta)}{\partial \theta}\right]}{\mathrm{E}[\mathrm{K} 1]}, \quad \text { a.s. }
$$

where $\mathrm{K} 1$ is the number of firings of any transition in the first cycle.

Q.E.D.

\subsection{Evaluation of $\Delta \pi^{1}\left(\mathrm{M}_{0}, \mathrm{p}\right)$ by IPA}

Let us apply the above results to evaluate the derivatives $\Delta \pi^{1}\left(M_{0}, \tau\right)$ needed in the heuristic algorithm presented in Section 5. 
Consider a family of stochastic timed event graphs $\operatorname{STEG}(\theta)=\left(\mathrm{N}, \mathrm{M}_{0},\left\{\mathrm{X}_{t}(\mathrm{k}, \theta)\right\}\right)$ where the transition firing times $X_{t}(k, \theta)=f_{t}\left(z_{t}(k), \theta\right)$ and the mappings $f_{t}(\bullet, \bullet)$ are defined as follows :

$$
f_{\tau}\left(z_{\tau}(k), \theta\right)=X_{\tau}(k, 0)+\theta \text { and } f_{t}\left(z_{t}(k), \theta\right)=X_{t}(k, 0) \text { for all } t \neq \tau
$$

Assuming that $X_{t}(k, 0)=X_{t}(k)$ for all $t$. From the definition of $\Delta \pi^{1}\left(M_{0}, \tau\right)$ (see (16)), it holds that :

$$
\pi\left(\mathrm{M}_{0}\right)=\pi\left(\mathrm{M}_{0}, 0\right) \quad \text { and } \quad \Delta \pi^{1}\left(\mathrm{M}_{0}, \tau\right)=\left.\lim _{\mathrm{k} \rightarrow \infty} \frac{\partial \pi\left(\mathrm{M}_{0}, \theta\right)}{\partial \theta}\right|_{\theta=0} .
$$

Remark also that in this particular case, :

$$
\mathrm{f}_{\mathrm{t}}^{\prime}\left(\mathrm{z}_{\mathrm{t}}(\mathrm{k}), \theta\right)=\frac{\partial \mathrm{f}_{\mathrm{t}}\left(\mathrm{z}_{\mathrm{t}}(\mathrm{k}), \theta\right)}{\partial \theta}= \begin{cases}1, & \text { if } \mathrm{t}=\mathrm{p} \bullet \\ 0, & \text { otherwise }\end{cases}
$$

which implies that condition (A3) always holds.

From Properties 12, 13, 14 and 15, we have:

Property 16.

Under some regular conditions,

$$
\Delta \pi^{1}\left(\mathrm{M}_{0}, \tau\right)=\left.\lim _{\mathrm{k} \rightarrow \infty} \frac{\partial \mathrm{S}_{\mathrm{t}}(\mathrm{k}, \theta)}{\partial \theta}\right|_{\theta=0} / \mathrm{k}, \quad \text { a.s. } \forall \mathrm{t} \in \mathrm{T}
$$

where

$$
\frac{\partial \mathrm{S}_{\mathrm{t}}(\mathrm{k}, \theta)}{\partial \theta}=1\{\tau(\mathrm{t}, \mathrm{k}, \theta)=\tau\}+\frac{\partial \mathrm{S}_{\tau(\mathrm{t}, \mathrm{k}, \theta)}\left(\mathrm{k}-\mathcal{M}_{0}(\rho(\mathrm{t}, \mathrm{k}, \theta)), \theta\right)}{\partial \theta}
$$

From this theorem, the derivatives $\Delta \pi^{1}\left(\mathrm{M}_{0}, \tau\right)$ can easily be evaluated along the simulation of step 3 of the algorithm 1 by using equations (23) and (24).

\section{NUMERICAL RESULTS}

This section is devoted to numerical examples. We first present results of the illustrative example introduced in Section 3.1. Results of randomly generated examples are then provided and compared with results of an alternative, simple algorithm.

\subsection{The illustrative example}

Consider the example introduced in Section 3.1. In this example, $C^{*}=10$ and we choose to reach a given cycle time $C=10.001$. Let us use the heuristic algorithm presented in Section 5. 
The optimal solution to the deterministic problem consists of putting one token in each of the places $p_{2}, p_{3}, p_{4}, p_{6}, p_{9}$ and $p_{10}$ and the $p$-invariant criterion value is equal to 7 . That is $\mathrm{M}_{0}=(0,1,1,1,0,1,0,0,1,1)^{\mathrm{T}}$.

In the second phase, simulations of the stochastic timed event graphs are performed. We use evolution equation (1) to simulate the system and long simulations $(\mathrm{K}=$ 20000) are performed to guarantee the precision.

In the first iteration of the second phase, the following data are obtained :

$$
\begin{aligned}
& \pi\left(\mathrm{M}_{0}\right)=12.476 \\
& \Delta \pi^{1}\left(\mathrm{M}_{0}, \mathrm{t}_{1}\right)=0.354, \Delta \pi^{1}\left(\mathrm{M}_{0}, \mathrm{t}_{2}\right)=0.520, \Delta \pi^{1}\left(\mathrm{M}_{0}, \mathrm{t}_{3}\right)=0.041 \\
& \Delta \pi^{1}\left(\mathrm{M}_{0}, \mathrm{t}_{4}\right)=0.417, \Delta \pi^{1}\left(\mathrm{M}_{0}, \mathrm{t}_{5}\right)=0.041, \Delta \pi^{1}\left(\mathrm{M}_{0}, \mathrm{t}_{6}\right)=0.125 \\
& \Pi\left(\mathrm{p}_{1}, \infty\right)=0.882, \Pi\left(\mathrm{p}_{2}, \infty\right)=0.621, \Pi\left(\mathrm{p}_{3}, \infty\right)=0.000, \Pi\left(\mathrm{p}_{4}, \infty\right)=0.000, \Pi\left(\mathrm{p}_{5}, \infty\right)=0.999, \\
& \Pi\left(\mathrm{p}_{6}, \infty\right)=0.929, \Pi\left(\mathrm{p}_{7}, \infty\right)=0.303, \Pi\left(\mathrm{p}_{8}, \infty\right)=0.697, \Pi\left(\mathrm{p}_{9}, \infty\right)=0.379, \Pi\left(\mathrm{p}_{10}, \infty\right)=0.504 \\
& W\left(\mathrm{p}_{1}, \infty\right)=0.882, \mathrm{~W}\left(\mathrm{p}_{2}, \infty\right)=0.621, W\left(\mathrm{p}_{3}, \infty\right)=0.000, \mathrm{~W}\left(\mathrm{p}_{4}, \infty\right)=0.000, \mathrm{~W}\left(\mathrm{p}_{5}, \infty\right)=0.999, \\
& W\left(\mathrm{p}_{6}, \infty\right)=0.929, \mathrm{~W}\left(\mathrm{p}_{7}, \infty\right)=0.303, W\left(\mathrm{p}_{8}, \infty\right)=0.697, W\left(\mathrm{p}_{9}, \infty\right)=0.379, W\left(\mathrm{p}_{10}, \infty\right)=0.504
\end{aligned}
$$

The approximate estimates of $h_{U}\left(M_{0}, p\right)$ are the following ones :

$$
\begin{aligned}
& h_{U}\left(M_{0}, p_{1}\right)=0.882, h_{U}\left(M_{0}, p_{2}\right)=1.905, h_{U}\left(M_{0}, p_{3}\right)=0.000, h_{U}\left(M_{0}, p_{4}\right)=0.000, h_{U}\left(M_{0}, p_{5}\right)=0.507, \\
& h_{U}\left(M_{0}, p_{6}\right)=0.004, h_{U}\left(M_{0}, p_{7}\right)=0.114, h_{U}\left(M_{0}, p_{8}\right)=0.617, h_{U}\left(M_{0}, p_{9}\right)=0.134, h_{U}\left(M_{0}, p_{10}\right)=1.288
\end{aligned}
$$

We choose to add a token in place $p_{2}$. The different iterations of the second phase are given in table 1 . Finally we obtain the optimal marking $\mathrm{M}_{0}=(0,6,1,2,1,1,0,1,1,1)^{\mathrm{T}}$.

Table 1: Steps of the second phase

\begin{tabular}{|c|c|c|c|}
\hline Iterations & Add one token in & Mean cycle time & Value of the criterion \\
\hline 1 & $\mathrm{p}_{2}$ & 10.912 & 8 \\
2 & $\mathrm{P} 5$ & 10.903 & 9 \\
3 & $\mathrm{P} 2$ & 10.421 & 10 \\
4 & $\mathrm{p} 4$ & 10.209 & 11 \\
5 & $\mathrm{P} 8$ & 10.132 & 12 \\
6 & $\mathrm{P} 2$ & 10.030 & 13 \\
7 & $\mathrm{P} 2$ & 10.008 & 14 \\
8 & $\mathrm{p} 2$ & 10.000 & 15 \\
\hline
\end{tabular}

\subsection{Additional numerical examples}

We finally present more numeral results for randomly generated stochastic timed event graphs.

These examples are generated as follows. First, a strong connected event graph is generated. The mean transition firing times are generated from a random variable uniformly distributed on $(0,10)$. For each transition, its firing times may be constant or 
follow one of the following distributions : exponential, normal, uniform, Erlang, Bernoulli.

The $\mathrm{p}$-invariant criterion is the summation of all elementary p-invariants and we choose to reach a given cycle time $\mathrm{C}=\mathrm{C}^{*}+0.1$.

The heuristic algorithm (Algorithm 1) is applied to all these examples and are compared with a naive algorithm (Algorithm 2). Algorithm 2 proceeds almost in the same manner as Algorithm 1 except that in the second phase, it uses the following criterion to choose the place in which the new token is added :

$$
\text { maximizes } g_{U}\left(M_{0}, p\right)=s\left(M_{0}, p\right) / u_{p}
$$

where $s\left(\mathrm{M}_{0}, \mathrm{p}\right)$ is the probability that place $\mathrm{p}$ is empty and its downstream transition is idle. Intuitively, adding a token in a place which is often empty may increase the throughput of the system. Algorithm 2 is based on this idea.

Table 2. Results of 20 numerical examples

\begin{tabular}{|c|c|c|c|c|c|}
\hline Ex. & $|T|$ & $|\mathrm{P}|$ & deterministic & $\begin{array}{c}\text { Criterion value } \\
\text { Algorithm 1 }\end{array}$ & Algorithm 2 \\
\hline 1 & 3 & 4 & 3 & 5 & 5 \\
\hline 2 & 18 & 34 & 495 & 812 & 958 \\
\hline 3 & 15 & 26 & 96 & 108 & 365 \\
\hline 4 & 10 & 19 & 49 & 50 & 50 \\
\hline 5 & 9 & 13 & 28 & 46 & 71 \\
\hline 6 & 6 & 9 & 9 & 10 & 11 \\
\hline 7 & 7 & 14 & 26 & 60 & 88 \\
\hline 8 & 9 & 18 & 81 & 145 & 485 \\
\hline 9 & 4 & 6 & 8 & 13 & 28 \\
\hline 10 & 5 & 10 & 20 & 44 & 46 \\
\hline 11 & 7 & 13 & 14 & 23 & 74 \\
\hline 12 & 19 & 39 & 747 & 766 & 73 \\
\hline 13 & 11 & 16 & 23 & 78 & 87 \\
\hline 14 & 6 & 8 & 7 & 8 & 90 \\
\hline 15 & 11 & 17 & 30 & 74 & $* 17128$ \\
\hline 16 & 29 & 56 & 8561 & 13326 & $* 404$ \\
\hline 17 & 20 & 38 & 257 & 287 & $* 17193$ \\
\hline 18 & 35 & 70 & 13129 & 15363 & $* 775$ \\
\hline 19 & 20 & 37 & 427 & 488 & $* 71$ \\
\hline 20 & 29 & 53 & 646 & 690 & \\
\hline & & & & & \\
\hline
\end{tabular}

Table 2 includes results of 20 examples. In this table, ${ }^{*}$ indicates the cases in which $C$ is not reached after 30 iterations and the criterion value corresponds to the $p$-invariant 
value of the marking obtained at the 30-th iteration. The cases in which the solution to the deterministic case remains optimal are not included.

From these results, algorithm 1 seems to provide near optimal solutions. In number of cases, algorithm 2 is not able to identify the adequate places to add tokens in.

\section{CONCLUSION}

In this paper, we have investigated the marking optimization problem of stochastic timed event graphs which consists of obtaining a given cycle time while minimizing a p-invariant criterion.

Some important properties have been established. In particular, we show that mutually reachable markings have the same average cycle time and the same $\mathrm{p}$ invariant criterion value. It was shown that the average cycle time is non-increasing with respect to the initial marking while the p-invariant criterion is non-decreasing. We further prove that the criterion value of the optimal solution is non-increasing in transition firing times in the stochastic ordering's sense.

Based on some existing lower bounds and upper bounds of the average cycle time, we show that the p-invariant criterion reaches its minimum when the firing times become deterministic and we establish the reachability conditions of a given cycle time.

We also proposed a heuristic algorithm. It starts from the optimal solution to the deterministic case and iteratively adds tokens to adequate places as long as the given cycle time is not obtained. Infinitesimal perturbation analysis of the average cycle time with respect to the transition firing times is used to identify the adequate places in which the new tokens are added. Numerical results show that the heuristic algorithm is efficient in providing near optimal solutions for non-trivial problems.

Further research directions are multiple. First an adaptative simulation length can be introduced to reduce to computation time. As can be noticed, the simulation length can be made very short when the average cycle time is still far from the given one and it should be long as the given cycle time is approached. The second research direction concerns the case in which the number of tokens to be added is important. Two solutions to this case, the first one consists of adding a variable number of tokens at each iteration and the second solution consists of approximating the tokens by a continuous flow which yields a continuous (or hybrid) Petri net model. The third research direction includes the extensions to other Petri net models representing various applications. 


\section{BIBLIOGRAPHY}

[1] F. BACCELLI, "Ergodic Theory of Stochastic Petri Networks", Annals of Probability, Vol. 20, No. 1, pp. 350-374, 1992.

[2] F. BACCELll and P. KONSTANTOPOUlOS, "Estimates of Cycle Times in Stochastic Petri Nets", Research Report No. 1572, INRIA, France, December 1991.

[3] F. BACCELlI and Z. LIU, "Comparison Properties of Stochastic Decision Free Petri Nets", to appear in IEEE Transactions on Automatic Control, 1992.

[4] J. CAMPOS, G. CHIOLA and M. SILVA, "Properties and Performance Bounds for Closed Free Choice Synchronized Monoclass Queueing Networks", IEEE Transactions on Automatic Control, Vol. 36, No. 12, pp. 1368-1382, December 1991.

[5] P. CHRETIENNE, "Les réseaux de Petri temporisés", Université Paris VI, Paris, France, Thése d'Etat, 1983.

[6] F. COMMONER, A. HOLT, S. EVEN and A. PNUELI, "Marked Directed Graphs", Journal of Computer and System Science, Vol. 5, No. 5, pp. 511-523, 1971.

[7] P. GLASSERMAN and D.D. YAO, "Algebraic Structure of Some Stochastic Discrete Event Systems, with Applications", Journal of Discrete Event Dynamic Systems: Theory and Applications, Vol. 1, No. 1, pp. 7-35, May 1991.

[8] P.J. HAAS and G.S. SHEDLER, "Stochastic Petri Nets: Modeling Power and Limit Theorem", Probability in Engineering and Informational Sciences, Vol. 5, pp. 477498, 1991.

[9] H.P. HILLION and J.M. PROTH, "Performance Evaluation of Job-Shop Systems Using Timed Event-Graphs", IEEE Transactions on Automatic Control, Vol. 34, No. 1, pp. 3-9, January 1989.

[10] Y.C. HO, "Performance Evaluation and Perturbation Analysis of Discrete Event Dynamic Systems", IEEE Transactions on Automatic Control, Vol. AC-32, No. 7, pp. 563-572, 1987. 
[11] S. LAFTIT, J.M. PROTH and X.L. XIE, "Event Graphs to Model and Evaluate Assembly Systems with Random Processing Times", Proc. of First International Conference on Automation Technology, Taipee, Taiwan, 1990.

[12] S. LAFTIT, J.M. PROTH and X.L. XIE, "Optimization of Invariant Criteria for Event Graphs", IEEE Transactions on Automatic Control, Vol. 37, No. 5, pp. 547555, May 1992.

[13] T. MURATA, "Petri Nets: Properties, Analysis and Applications," Proceedings of the IEEE, vol. 77, N4, pp. 541-580, April 1989.

[14] J.M. PROTH, N. SAUER and X.L. XIE, "Stochastic Timed Event Graphs : Bounds, Cycle Time Reachability and Marking Optimization", Research Report No. 1807, INRIA, France, 1993.

[15] J.M. PROTH and X.L. XIE, "Performance Evaluation and Optimization of Stochastic Timed Event Graphs", submitted for publication in IEEE Transactions on Automatic Control, June 1992.

[16] C.V. RAMAMOORTHY and G.S. HO, "Performance Evaluation of Asynchronous Concurrent Systems using Petri Nets", IEEE Trans. Software Eng., Vol. SE-6, No. 5, pp. 440-449, 1980.

[17] J.A.C. RESING, R.E. de VRIES, G. HOOGHIEMSTRA, M.S. KEANE and G.J. OLSDER, "Asymptotic Behaviour of Random Discrete Event Systems", Stochastic Processes and their Applications, Vol. 36, pp. 195-216, 1990.

[17] S.M. ROSS, "Stochastic Processes", J. Wiley \&Sons, New York, 1983.

[19] N. SAUER and X.L. XIE, "New Performance Bounds and Asymptotic Properties of Stochastic Timed Event Graphs", Research Report No. 1826, INRIA, France, also submitted to the IEEE Transactions on Automatic Control, 1992.

[20] N. SAUER and X.L. XIE, "Marking Optimization of Stochastic Timed Event Graphs", submitted to the 14th Int. Conf. on Applications and Theory of Petri Nets, Chicago, June 1993.

[21] V.M. SAVI and X.L. XIE, "Liveness and Boundedness Analysis for Petri Nets with Event Graphs Modules", in Application and Theory of Petri Nets 1992, K. Jensen (ed.), Lecture Notes of Computer Science, Springer Verlag, pp. 328-347, 1992. 
[22] M. SILVA, "Petri Nets and Flexible Manufacturing," in Advances in Petri Nets 1989, G. Rozenberg (ed.), Lecture Notes of Computer Science, Springer Verlag, pp. $374-417,1989$.

[23] D. STOYAN, "Comparison Methods for Queues and Other Stochastic Models", English translation (D.j. Daley editor), J. Wiley and Sons, New York, 1984.

[24] R. SURI, "Perturbation Analysis: The State of the Art and Research Issues Explained via the GI/G/1 Queue", Proceedings of the IEEE, Vol. 77, No. 1, pp. 114137, January 1989.

[25] X.L. XIE, "Superposition Properties and Performance Bounds of Stochastic Timed Event Graphs", Research Report No. 1807, INRIA, France, also submitted to IEEE Trans. on Automatic Control, 1992

[26] X.L. XIE, "On the Impact of Randomness in Production Lines Controlled by Kanbans", To appear in the Proceedings of the Second European Control Conference (ECC 93), Groningen, The Netherlands, June 1993 


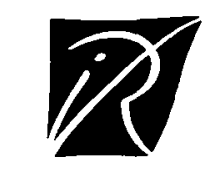

Unité de Recherche INRIA Lorraine

Technopôle de Nancy-Brabois - Campus Scientifique

615, rue du Jardin Botanique - B.P. 101 - 54602 VILLERS LES NANCY Cedex (France)

Unité de Rccherche INRIA Rennes IRISA. Campus Universitaire de Beaulieu 35042 RENNES Ccdex (France)

Unité de Recherche INRIA Rhône-Alpes 46, avenue Félix Viallet - 3803I GRENOBLE Cedex (France)

Unité de Recherche INRIA Rocquencourt Domaine de Voluceau - Rocquencourt - B.P. 105 - 78153 LE CHESNAY Cedex (France)

Unité de Recherche INRIA Sophia Antipolis 2004, route des Lucioles - B.P. 93 - 06902 SOPHIA ANTIPOLIS Cedex (France)

\section{EDITEUR}

INRIA - Domaine de Voluceau - Rocquencourt - B.P. 105 - 78153 LE CHESNAY Cedex (France)

ISSN $0249-6399$ 